http://dx.doi.org/10.21611/qirt.1994.011

\title{
Rescaling of thermographic camera readouts based on the results of contact measurements during unsteady process
}

by PRĘGOWSKI P. *

^PIRS - PREGGOWSKI INFRARED SERVICES; ul. Ząchodzacego Storica 36, 01 - 495 Warsaw, Poland

\section{Abstract}

This paper gives practical advice based on experience gained by the author's company during the investigations of soldering in the vacuum chamber with the use of extemal and contact type reference sources. The conditions of these measurements were far from optimum ones. The main elements of the applied simple procedures are discussed.

\section{Introduction}

The purpose of our study was to help in establishing the best shapes of soldered surfaces of metal and ceramic elements as well as to give the proper rates of heating and cooling. It was done in order to avoid too strong thermal stresses and breaks resulting from differences in thermal characteristics of these materials.[1,2]

Object of measurements was pressed by inductively heated graphite stamps. Thermographic camera was situated outside of the vacuum chamber. In a case of additional window in the optical path between camera and a measured object, a further calibration of the system is required [3].

In the presented case, the radiant properties of the vacuum chamber's window have been changed very little. The IR camera settings were changed in the large range. We used experimental calibration procedure based on the extemal radiant source with the temperature being determined with the thermocouple. This source, being our reference, was chosen as a part of measured field of the object of measurements.It is a very popular method used when the temperatures are stable enough and attachment of the contact sensor does not change the measurement conditions.

In case of thermally unsteady processes, additional problems should be considered. One of them results from differences between dynamics of the method of infrared measurements and method of measurement by means of contact sensor. Many various reasons cause that temperature indications of the contact methods have been delayed in comparison with the radiant ones. Such an effect is easy to observe only in the case of two way process i.e. heating and cooling which are typical for e.g. soldering or hardening.

The paper shows the consequences of the differences in the applied contact and radiation methods. The measuring set together with the main elements of the applied procedure for thermoprofiles scaling are presented below. 


\section{Conditions of measurements}

The general measuring system and object of investigations as well as attachment of thermocouples for reference temperature determination are shown in figure 1.

Temperature of the metal-ceramic object was being changed from ambient temperature up to $1100 \mathrm{~K}$. Radiation of this object was transmitted through the quartz window. These investigations were carried out using the old-type thermographic camera (AGA 680) with analog-direct coupling processing, image and line-profile displays. Although, at present, we use PTRWIN from CEDIP -and our work is easier and more precise -but necessity of special procedures often exist.

Spectral characteristics of the camera and quartz window as well as resulting effective characteristic for the measured signals are shown in figure 2. Some of results i.e. informative parts of thermoprofiles for three phases of soldering are shown in figure3.

Important elements for quantitative aspects of these surveys were:

a) long duration of experiment( $>90 \mathrm{~min}$, problems of camera drifts),

b) strong influence of quartz glass window,

c) radiant properties of measured surfaces (300K-1100K range, emissivity differences, of metal and ceramics, angles dependence),

d) delay time of the contact type measurements used as reference signal to real-time answer of IR thermography,

e) form of presentation of measurement results.

Short remarks on our choices and results are as follows:

Camera drifts and its internal radiation:

Problem of drifts was minimized using a stable external radiant source before any series of measurements for "zeroing" of the camera readouts and checking the gain changes. It has been done for the largest aperture of camera optics and the highest gensitivity settings.

\section{Vacuum chambers window:}

Spectral characteristic of the chambers window is shown in figure 2. An optimum upectral filtering was impossible to apply during these investigations. In result, the quartz glass acted as a low wavelength-pass filter or as a window but simultaneously as a good emitter for wavelengths longer than 3.9 micrometers.

The problem of emission was checked and found as being not very important. For example in a case of the signals from blackbody (!) with temp. of $340 \mathrm{~K}$, what was the highest expected temperature of this chamber's window, the output signal for f/number 5. 1 was about 9 isotherm units, when signal from 1100K was about 400 isotherm units. Based on the previous experiment, insignificant changes of effective transmission have been assumed, too. For lowering of above mentioned effects, cooling of window frame is recommended.

\section{Emissivity and thermal data of the measured surfaces:}

Maximum temperature of the process reached more than $1100 \mathrm{~K}$. Very significant differences of the emission coefficients were minimized by covering the appropriate narrow stripe of metal and ceramic surfaces with the soot. The width of this strip was only as large as it was needed according to the camera geometrical resolution. In these conditions only the emission component of the total signal was considered. 
http://dx.doi.org/10.21611/qirt.1994.011

Metals and ceramics have also significant differences in the thermal resistances. Significantly lower resistance has steel.

\section{Contact type temperature sensors:}

PtRh10 thermocouples, unfortunately a little too thick, were bonded to the two parts of the soldered object. The reference field was chosen on the steel surface. According to this choice new sources of the errors have been considered :

-differences between the real surface temperature at a field of reference sample ( within the zone of thermocouple contact with the object and the thermocouple thermometer indications),

-assignation of the reference area i.e. thermocouple zone to the corresponding position on the profile thermogram,

- readouts and position errors of the measured reference point at the profilogram.

\section{Calibration}

Experimental calibration based on reference radiation of the part of measured surface was applied.The idea of calibration procedure was based on the assumption, that for many points, within the range of temperature changes, simultaneously thermocouple indications and adequate camera readings are noted.

In our case, the data of longitudinal temperature changes were expected and the absolute temperatúre was of secondary meaning. The objective lens apertures (1.8-5.1) as well as few positions of sensitivity settings (100-500) were used. In such a case, two ways for comparison of the measured quantities are possible:

a) in a very narrow range - between the same aperture and sensitivity settings,

b) in other cases - after special correction for normalization.

\section{Procedure for finding the normalized values of the output signals:}

In order to realize the way b) we have applied the next procedure:

-for the given temperature of the stable blackbody,

-we checked experimentaly the theoretical connections between apertures (finding that changes of the radiant power in a few cases were not exactly equal to the second power of f/number ratios),

-similary we have checked the changes between settings of various sensitivities (finding expected values),

-finally we have used the following formula for normalization, mainly for the conditions of settings during the highest temperature measurements:

$$
j(y)=\left(y \cdot S / W+j_{0} \cdot f_{0}^{2} / f^{2}\right) \cdot k \cdot f^{2} / f_{x}^{2}
$$

-where:

$j(y)$ - quantity of normalized units,

y - quantity of units from measurement,

s - sensitivity during measurement,

w - factor of thermoprofile scaling,

$j_{0}$ - offset units,

$f_{x}$ - normalized f/number,

$f-f /$ number during measuremets,

$f_{0}-f$ /number $(=1.8)$ for offset setting

$k$ - factor for correction f/number relations, 
http://dx.doi.org/10.21611/qirt.1994.011

\section{Procedure for finding calibration chart:}

The calibration procedure seemed to be extremely simple because of many important simplifications (soot coverages, external reference being a part of the measured object). In practice it was not so simple to obtain adequate level of the precision. The below procedure for analytical determination of calibration chart has been evaluated.

During the experiment the following data have been noted:

time, offset level for drift correction, aperture and sensitivity settings, temperature from thermocouple (in steps for every $50 \mathrm{~K}$ ) together with equivalent readouts from IR camera.

Next, during the interpretation phase, the following steps have been executed:

normalization of the isotherm unit readouts from the area of the reference - according to (1),

drawing the initial "calibration charts" - i.e. values of normalized isotherm units "j(y,s,f,jo)" plotted against temperature indicated by thermocouple,

For all cases these curves were different for the heating and cooling phases. It was shown in figure4.

The hysteresis effect,considered together with the measurement conditions, indicates that these differences are dependent on the rate of the temperature changes.

determination of gradients of the temperature changes and drawing the temperature against experiment duration.

Il was determined that the rate of heating (max. 2.6K/s) was 3 to 5 times higher than for cooling phase.

adetermination of points for drawing the final calibration chart with the weighted method.

The idea of this method was to place the chosen calibration point between two curves, so many times near to "cooling curve" than for "heating curve", as many times rate of heating was higher than rate for cooling for reaching the same values of camera readout.

\section{Procedure for tables obtaining for scaling thermoprofiles:}

In order to set tables with the units of thermoprofile and corrected temperature of an object, the MathCAD software was applied. Although other mathematical expressions for calibration curve are known [4,5] more simpler formulas (2) and (3) have been applied:

$$
j(T)=a \cdot \exp (b / T)
$$

where:

$$
b=\ln \left[j_{2} \cdot T_{1} \cdot T_{2} / j_{1} \cdot\left(T_{1}-T_{2}\right)\right]
$$$$
a=j_{1} \cdot \exp \left(-b / T_{1}\right)
$$

Important advantage of this method is possibility to express this function basing only on two different readouts $\left(j_{1}, j_{2}\right)$ with adequate temperatures $\left(T_{1}, T_{2}\right)$ in $[K]$. Finding the proper points for above approximation is extremely important. In practice, it is convenient only when all the curve is drawn [6]. The whole procedure is very simple and the obtained results, very often, are satisfactory. 
http://dx.doi.org/10.21611/qirt.1994.011

Now, based on the formulas (1) and (2) the final expression for scaling the thermoprofiles can be found as:

$$
T(y)=b / \ln (j(y) / a)
$$

where:

$$
b=\ln \left[j_{2} \cdot T_{1} \cdot T_{2} / j_{1} \cdot\left(T_{1}-T_{2}\right)\right] \quad a=j_{1} \cdot \exp \left(-b / T_{1}\right)
$$

In such a way tables for convenient scaling of ordinates of the thermoprofiles were fulfilled.

Equations (2),(3) can be used, also, for specialized thermographic software working in the mode of the "experimental calibration". We sometimes use such an option with the CEDIP's PTRWIN for other tasks.

\section{Conclusions}

In the presented case the very simple procedures were satisfactory. The special care and adequate experimental and analytical procedures are needed for scaling camera readouts during the thermally unsteady processes, especially when the methods of various dynamic characteristics are applied.

\section{Acknowledgement}

Author would like to thank Mr W. Adamczewski and Mr.D.Golański for their valuable assistance for realization of the described investigations.

\section{REFERENCES}

[1] PREGGOWSKI (P), ADAMCZEWSKI (W)- Thermographic investigation of soldering process, PIRS report ITB/44, November 1992

[2] GOLAŃSKI (D), - Application, of the thermography during investigations of the vacuum process of the metal-ceramic soldering, proceedings of the First National Conference on the IR Thermography and Radiometry, Szczyrk, November, 1992, p.179-182, ODKT

[3] RYU (Z,M), PEARCE (J.A)- Thermographic calibration of graybody objects, SPIE, vol.1313, 1990, p.282-289

[4] MEDDINGS (T.J.), WILSON (C.W.)- Application of a commercially available thermal imager to a film cooled panel temperature measurement,SPIE vol.1320,1990,p.262-273 [5] WARREN (C), Digital analysis of infrared imagery", SPIE, vol.246, 1980, p.144-151 [6] PREGGOWSKI [P]- The analyses of radiant signal marks and their influence for the thermographic device designing, doctor thesis, Military University of Technology, Warsaw, 1985 

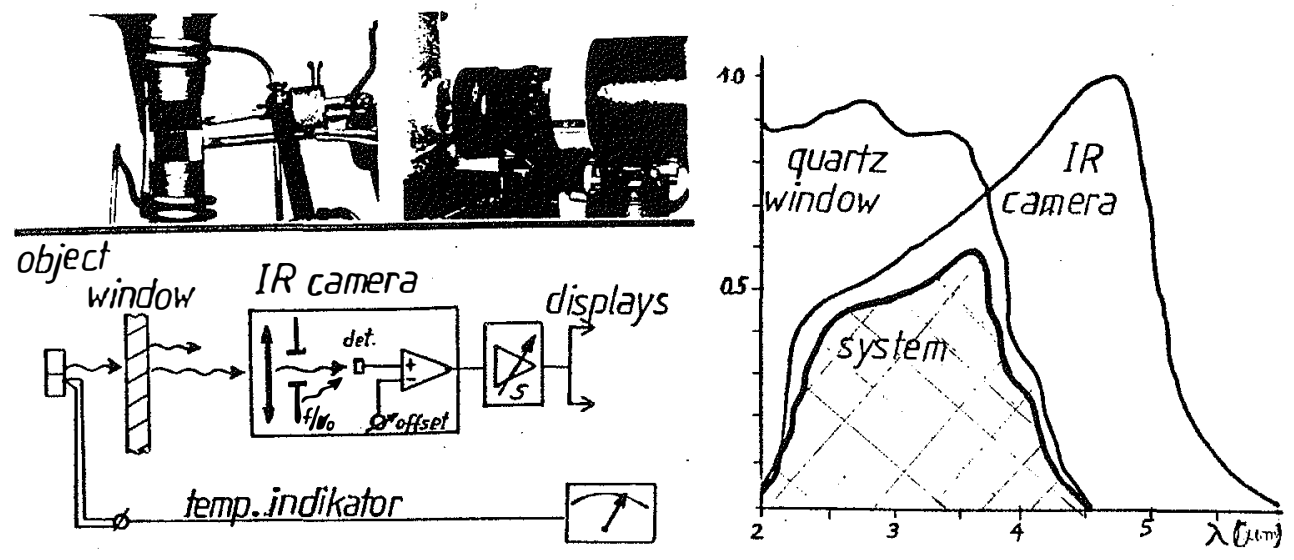

Fig.1. Scheme of measuring set and thermocouples attachment

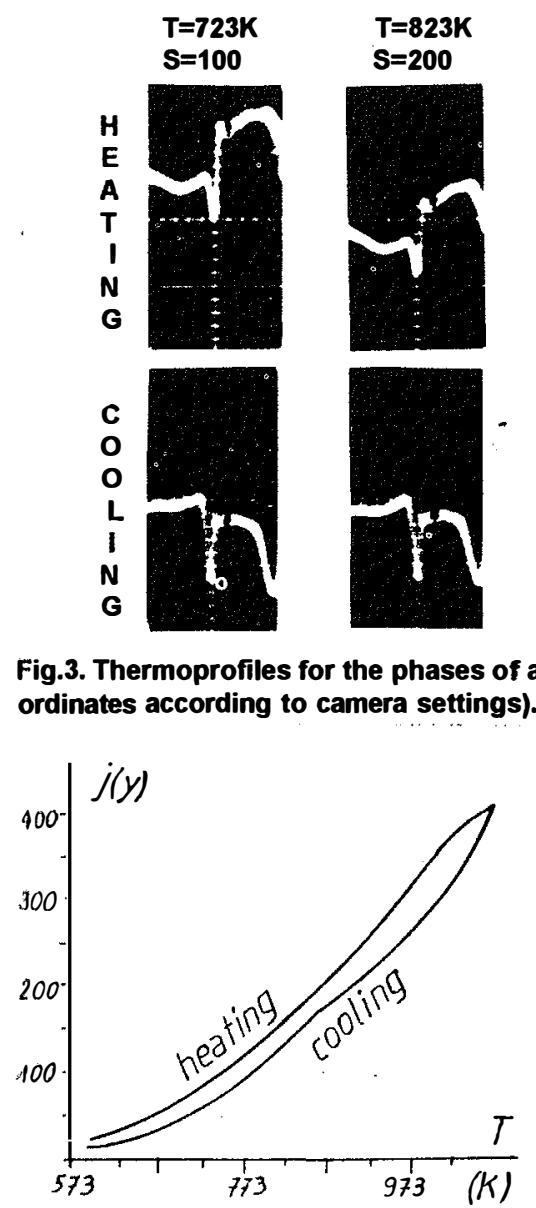
ordinates according to camera settings).
Fig.4 Initiaition -"calibration curves"

-after experiment and normalization
Fig.2. Influence of the quartz window of vacuum chamben(spectral charaiteristics)

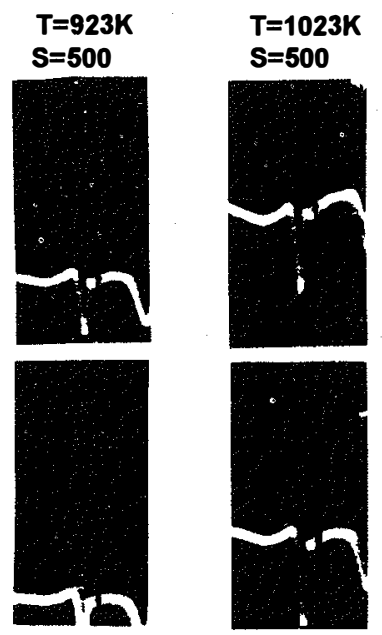

$$
\begin{gathered}
T=1073 k \\
S=500
\end{gathered}
$$

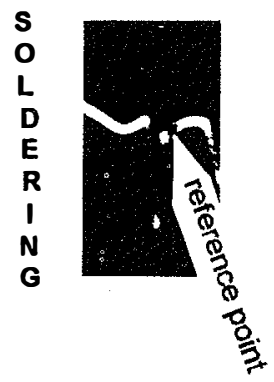

Fig.3. Thermoprofiles for the phases of a) heating, b) soldering, c) cooling ( various scales of

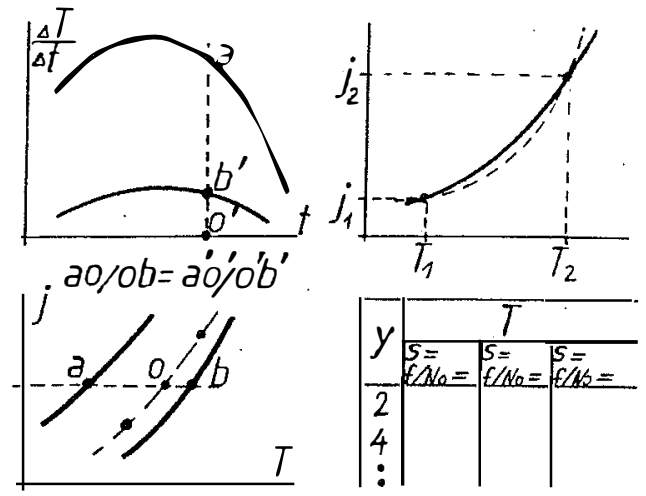

Fig. 5 Idea of the corrected curve establishing 